\title{
PREPARACIÓN Y CARACTERIZACIÓN DE BIOSORBENTES BASADOS EN COMPÓSITOS DE MWCNT-MAGNETITA- HIDROXIAPATITA Y SU POTENCIAL APLICACIÓN EN LA REMOCIÓN DE METALES PESADOS
}

\author{
Golfer Muedas Taipe ${ }^{* a}$, Andrea Leticia Almandoz Landa ${ }^{a}$, \\ Fátima Amanda Santillán Espinoza ${ }^{\mathrm{a}}$
}

\begin{abstract}
RESUMEN
En el presente estudio, se prepararon nuevos biosorbentes basados en compósitos de magnetita $\left(\mathrm{Fe}_{3} \mathrm{O}_{4}\right)$, hidroxiapatita (HAp) y nanotubos de carbono de pared múltiple (MWCNT). Los materiales preparados fueron caracterizados mediante técnicas de dispersión dinámica de luz (DLS) espectroscopía infrarroja (FTIR), microscopía electrónica de barrido (SEM), magnetometría de muestra vibrante (VSM), espectroscopía Raman y voltamperometría de onda cuadrada. La magnetita sintetizada presentó un comportamiento ferromagnético. La HAp tuvo menor tamaño mediante la síntesis hidrotermal. Además, se evaluó el posible uso del compósito que involucraba a los tres materiales de partida $\left(\mathrm{Fe}_{3} \mathrm{O}_{4} / \mathrm{MWCNT} / \mathrm{HAp}\right)$ en la remoción de un metal pesado como $\mathrm{As}(\mathrm{V})$ en solución acuosa, teniendo en cuenta la influencia de factores como $\mathrm{pH}$, masa de adsorbente y tiempo de contacto. Se obtuvo los valores máximos de porcentaje de remoción de este metal (mayor a $85 \%$ ) a condiciones de $\mathrm{pH} 6$, dosis de adsorbente de $0,5 \mathrm{~g} \mathrm{~L}^{-1} \mathrm{y}$ un tiempo de contacto de $2 \mathrm{~h}$.
\end{abstract}

Palabras clave: compósitos, magnetita, hidroxiapatita, nanotubos de carbono de pared múltiple, remoción.

\section{PREPARATION AND CHARACTERIZATION OF BIOSORBENTS BASED ON MWCNT-MAGNETITE-HYDROXYAPATITE COMPOSITES AND ITS POTENTIAL APPLICATION IN THE REMOVAL OF HEAVY METALS}

\begin{abstract}
In the present study, new biosorbents based on magnetite $\left(\mathrm{Fe}_{3} \mathrm{O}_{4}\right)$, hydroxyapatite (HAp) and multiple-walled carbon nanotubes (MWCNT) composites were prepared. The materials were characterized by dynamic light scattering (DLS), infrared spectroscopy (FTIR), scanning electron microscopy (SEM), vibrating sample magnetometry (VSM), raman spectroscopy

\footnotetext{
${ }^{* a}$ Escuela Profesional de Química, Facultad de Ciencias, Universidad Nacional de Ingeniería, Av. Túpac Amaru 210, Lima 25, Perú. E-mail: gmuedast@uni.edu.pe
} 
and square wave voltammetry (SWV). The synthesized magnetite presented a ferromagnetic behavior. HAp was smaller by hydrothermal synthesis. In addition, the possible use of the composite involving the 3 starting materials (Fe3O4/MWCNT/HAp) in the removal of a heavy metal such as $\mathrm{As}(\mathrm{V})$ in aqueous solution was evaluated, taking into account the influence of factors such as $\mathrm{pH}$, adsorbent mass and contact time. The maximum values of percentage of removal of this metal (greater than $85 \%$ ) were obtained under conditions of $\mathrm{pH} 6$, adsorbent dose of $0.5 \mathrm{~g} \mathrm{~L}^{-1}$ and a contact time of 2 hours.

Key words: composites, magnetite, hydroxyapatite, multi-walled carbon nanotubes, arsenic, removal.

\section{INTRODUCCIÓN}

Los metales pesados son altamente perjudiciales para la salud humana ${ }^{1}$, incluso a bajas concentraciones debido a su bioacumulación. La variedad de técnicas y procesos de tratamiento químico desarrollados para eliminar a los metales pesados del agua contaminada incluyen principalmente a los procesos de: precipitación $^{2}$, intercambio de iones ${ }^{3}$, nanofiltración y ósmosis inversa ${ }^{4}$, sin embargo, la adsorción se presenta como una mejor alternativa debido a su bajo costo, alta eficiencia, baja probabilidad de formación de productos secundarios y una alta posibilidad de reuso de los materiales adsorbentes ${ }^{5}$.

El uso de partículas magnéticas es un recurso muy utilizado y atractivo por la facilidad de poder usarlos y recuperarlos mediante un campo magnético externo y por su comportamiento superparamagnético a temperatura ambiente con alta magnetización de saturación ${ }^{2}$, por ello, la magnetita está siendo usada en trabajas que involucran la remoción de metales pesados tóxicos provenientes de terrenos mineros o muy aledaños, que es una problemática actual y de gran interés ${ }^{6}$.

La hidroxiapatita ya antes ha sido utilizada en diferentes ámbitos como: producción de materiales fluorescentes ${ }^{7}$, portadores de fármacos ${ }^{8}$, aplicaciones en el campo de biomedicina ${ }^{9}$ y medioambiental ${ }^{10}$. Estos usos mencionados se producen debido a que posee ciertas propiedades como: alta estabilidad, compatibilidad ambiental, alta área superficial, actividad fotocatalítica ${ }^{11}$.

Por otra parte, los nanotubos de carbono (CNT), representan materiales de interés actual utilizados como complementos ${ }^{12}$ (recipientes con filtros basados en nanotubos de carbono) o en la elaboración de materiales aplicados al tratamiento de una amplia variedad de contaminantes debido a que las nanoestructuras de carbón presentes en ellos le conferirían una mayor superficie de contacto con el contaminante. Estudios revelan la preferencia de utilizar nanotubos de carbono de pared múltiple (MWCNT) sobre los de pared simple, debido a que estos últimos presentan fuertes tendencias hacia una aglomeración, lo cual disminuiría su efectividad como material adsorbente ${ }^{13}$. 
Finalmente, en este trabajo se ha realizado la síntesis de magnetita $\left(\mathrm{Fe}_{3} \mathrm{O}_{4}\right)$ recubierta con citrato, síntesis de la hidroxiapatita (HAp) y la preparación de diferentes biosorbentes (compósitos) basados en la combinación de los materiales de partida mencionados, con el fin de poder aplicarlas en la resolución y mejora de problemas relacionados con la calidad del agua.

\section{PARTE EXPERIMENTAL}

\section{Materiales}

Los reactivos $\mathrm{FeCl}_{3} \cdot 6 \mathrm{H}_{2} \mathrm{O}$ y $\mathrm{FeCl}_{2} \cdot 4 \mathrm{H}_{2}, \mathrm{NH} 4 \mathrm{OH}$ y citrato de sodio y $\mathrm{N}_{2}$ ultrapuro fueron utilizados en la síntesis de la magnetita. $\mathrm{El} \mathrm{Ca}\left(\mathrm{NO}_{3}\right)_{2} \cdot 4 \mathrm{H}_{2} \mathrm{O},\left(\mathrm{NH}_{4}\right)_{2} \mathrm{HPO}_{4}, \mathrm{NH}_{4} \mathrm{OH}, \mathrm{H}_{3} \mathrm{PO}_{4}$, $\mathrm{Ca}(\mathrm{OH})_{2}, \mathrm{Ca}\left(\mathrm{NO}_{3}\right)_{2} \cdot 4 \mathrm{H}_{2} \mathrm{O}, \mathrm{C}_{6} \mathrm{H}_{5} \mathrm{Na}_{3} \mathrm{O}_{7} \cdot 2 \mathrm{H}_{2} \mathrm{O}$ y Na $3 \mathrm{PO}_{4} \cdot 12 \mathrm{H}_{2} \mathrm{O}$ fueron utilizados por diversos métodos para la síntesis de la hidroxiapatita (HAp) y la preparación de los compósitos. Todos los reactivos químicos usados en las síntesis fueron de grado analítico y adquiridos en Merck Peruana S.A. Los nanotubos de carbono de pared múltiple (MWNCT), utilizados en este trabajo, son de uso comercial y fueron suministrados por Sigma-Aldrich Co., Ltd, Estados Unidos.

\section{Síntesis de magnetita mediante coprecipitación y estabilización con citrato ${ }^{2}$}

La magnetita fue sintetizada utilizando al $\mathrm{FeCl}_{3} \cdot 6 \mathrm{H}_{2} \mathrm{O}$ y $\mathrm{FeCl}_{2} \cdot 4 \mathrm{H}_{2} \mathrm{O}$ como precursores metálicos. La preparación consistió básicamente en la precipitación a partir de la mezcla de cloruros (relación molar $\mathrm{Fe}^{3+}: \mathrm{Fe}^{2+}$ de 2:1) añadiendo gota a gota $\mathrm{NH}_{4} \mathrm{OH}$ al $25 \%(10 \mathrm{~mL})$ en agitación continua. $\mathrm{El} \mathrm{NH}_{4} \mathrm{OH}$ fue utilizado como agente precipitante. La mezcla resultante se lavó cuatro veces con agua ultrapura, se dejó en reposo y finalmente se separó el precipitado de color negro (magnetita) utilizando un campo magnético externo de un imán. La magnetita obtenida se secó durante $1 \mathrm{~h}$ a $100{ }^{\circ} \mathrm{C}$. El agua utilizada para la preparación de la mezcla de cloruros y para el recubrimiento con citrato fue sometida a una atmósfera inerte de $\mathrm{N}_{2}$ durante $1 \mathrm{~h}$ a $25^{\circ} \mathrm{C}$. Para la estabilización con citrato, la magnetita se dispersó en $50 \mathrm{~mL}$ de agua ultrapura y se sometió a un baño de ultrasonido durante $5 \mathrm{~min}$. La dispersión obtenida se sometió a un baño María a $70{ }^{\circ} \mathrm{C}$, en esas condiciones, se añadió gota a gota $10 \mathrm{~mL}$ de citrato de sodio $0,1 \mathrm{M}$ y se dejó enfriar, manteniendo la agitación por $12 \mathrm{~h}$. La magnetita estabilizada se separó por decantación con ayuda de un imán. Finalmente, se hicieron lavados con agua ultrapura, hasta llevarlo a neutralidad y el material obtenido se secó en estufa.

\section{Preparación de hidroxiapatita mediante método químico ${ }^{14}$}

Se prepararon soluciones de $\mathrm{Ca}\left(\mathrm{NO}_{3}\right)_{2} \cdot 4 \mathrm{H}_{2} \mathrm{O} 0,33 \mathrm{M}$ y de $\left(\mathrm{NH}_{4}\right)_{2} \mathrm{HPO}_{4}$ 0,12 M. La solución de amonio se adicionó gota a gota sobre la solución de calcio bajo agitación continua durante $1 \mathrm{~h} \mathrm{a} 90{ }^{\circ} \mathrm{C}$. Luego de este tiempo, se añadió por goteo $5 \mathrm{~mL}$ de $\mathrm{NH}_{4} \mathrm{OH}$ al $25 \%$ y se dejó en reposo durante 10 días para la precipitación de la hidroxiapatita. El precipitado obtenido se separó por centrifugación, luego se lavó cuatro veces con agua ultrapura y se secó a $250{ }^{\circ} \mathrm{C}$ durante $1 \mathrm{~h}$. Finalmente, el precipitado se calcinó a $1000^{\circ} \mathrm{C}$ durante $3 \mathrm{~h}$. 


\section{Preparación de hidroxiapatita mediante método sol-gel ${ }^{15}$}

Se prepararon soluciones de $\mathrm{H}_{3} \mathrm{PO}_{4} 0,6 \mathrm{M}$ y $\mathrm{Ca}(\mathrm{OH})_{2}, 1,0 \mathrm{M}$, las cuales fueron mezcladas a una velocidad de $1,5 \mathrm{~mL} / \mathrm{min}$, en agitación continua durante $1 \mathrm{~h}$ a $90{ }^{\circ} \mathrm{C}$, luego de ello se agitó la mezcla durante $1 \mathrm{~h}$ adicional hasta completar la reacción. La mezcla resultante se dejó en reposo por $72 \mathrm{~h}$ para la precipitación, luego de ello, se procedió a lavar con abundante agua ultrapura hasta neutralidad $(\mathrm{pH}=7)$. Finalmente, el polvo de hidroxiapatita se llevó a la mufla a $1000{ }^{\circ} \mathrm{C}$ durante $3 \mathrm{~h}$.

\section{Preparación de hidroxiapatita mediante método hidrotermal ${ }^{16}$}

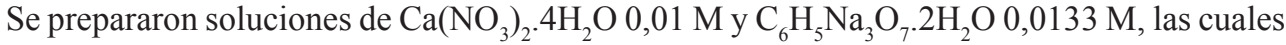
fueron mezclados en agitación constante durante $10 \mathrm{~min}$. Luego de ello, se añadió lentamente $15 \mathrm{~mL}$ de una solución de $\mathrm{Na}_{3} \mathrm{PO}_{4} \cdot 12 \mathrm{H}_{2} \mathrm{O} 0,006 \mathrm{M}$ bajo agitación vigorosa. La mezcla resultante se llevó a una autoclave de acero a $150^{\circ} \mathrm{C}$ durante $24 \mathrm{~h}$ (tratamiento hidrotermal) y el producto obtenido se centrifugó a $15000 \mathrm{rpm}$ durante $10 \mathrm{~min}$. El precipitado se lavó con agua ultrapura tres veces y finalmente se secó a $100^{\circ} \mathrm{C}$ durante $1 \mathrm{~h}$.

\section{Síntesis de compósito Fe304/HAp ${ }^{17}$}

Se preparó una solución acuosa de $1,85 \mathrm{mmol}$ de $\mathrm{FeCl}_{2} \cdot 4 \mathrm{H}_{2} \mathrm{O}$ y $37,0 \mathrm{mmol}$ de $\mathrm{FeCl}_{3} \cdot 6 \mathrm{H}_{2} \mathrm{O}$, a la cual se le agregó $12 \mathrm{~mL}$ de $\mathrm{NH}_{4} \mathrm{OH}$ bajo agitación constante. A esta mezcla se le agregó ácido cítrico $0,1 \mathrm{M}(\mathrm{pH}=10)$, bajo calentamiento de $90{ }^{\circ} \mathrm{C}$ y atmósfera inerte de $\mathrm{N}_{2}$. El sólido resultante fue obtenido mediante separación con un imán, fue lavado con agua ultrapura y se dejó en reposo durante $10 \mathrm{~min}$. Luego de ello, se adicionó $100 \mathrm{~mL}$ de una solución de $\mathrm{Ca}\left(\mathrm{NO}_{3}\right)_{2} \cdot 4 \mathrm{H}_{2} \mathrm{O}$ y $\left(\mathrm{NH}_{4}\right)_{2} \mathrm{HPO}_{4} 1: 1(\mathrm{pH}=11)$ en agitación continua durante $30 \mathrm{~min}$. La mezcla resultante se sometió a calentamiento a $90{ }^{\circ} \mathrm{C}$ durante $2 \mathrm{~h}$. Terminado este proceso, se dejó envejecer el producto por un periodo de $12 \mathrm{~h}$ a temperatura ambiente. Finalmente, el compósito obtenido se filtró varias veces con agua ultrapura hasta llegar a neutralidad y fue secado al vacío.

\section{Síntesis de compósito $\mathrm{Fe}_{3} \mathrm{O}_{4} / \mathrm{MWCNT}$}

Se dispersó $250 \mathrm{mg}$ de MWCNT en una solución acuosa de 1,85 mmol de $\mathrm{FeCl}_{2} \cdot 4 \mathrm{H}_{2} \mathrm{O}$ y 37,0 mmol de $\mathrm{FeCl}_{3} \cdot 6 \mathrm{H}_{2} \mathrm{O}$. Luego de ello, se agregó $12 \mathrm{~mL}$ de $\mathrm{NH}_{4} \mathrm{OH}$, bajo agitación constante; a la mezcla obtenida se le agregó $\mathrm{C}_{6} \mathrm{H}_{8} \mathrm{O}_{7} 0.1 \mathrm{M}(\mathrm{pH}=10)$ bajo atmósfera inerte de $\mathrm{N}_{2} \mathrm{y}$ en calentamiento a $90{ }^{\circ} \mathrm{C}$. El sólido resultante fue separado por decantación con ayuda de un imán y luego fue lavado con agua ultrapura.

\section{Síntesis de compósito $\mathrm{Fe}_{3} \mathrm{O}_{4} / \mathrm{MWCNT/HAp}$}

Para la preparación del compósito $\mathrm{Fe}_{3} \mathrm{O}_{4} / \mathrm{MWCNT} / \mathrm{HAp}$ se utilizó la $\mathrm{Fe}_{3} \mathrm{O}_{4} / \mathrm{MWCNT}$ preparado anteriormente. A este compósito se le añadió $100 \mathrm{~mL}$ de solución de $\mathrm{Ca}\left(\mathrm{NO}_{3}\right)_{2} \cdot 4 \mathrm{H}_{2} \mathrm{O}$ y $\left(\mathrm{NH}_{4}\right)_{2} \mathrm{HPO}_{4} 1: 1(\mathrm{pH}=11)$ bajo agitación constante durante $30 \mathrm{~min}$. La mezcla resultante se sometió a calentamiento a $90^{\circ} \mathrm{C}$ durante $2 \mathrm{~h}$. Una vez terminado el proceso se dejó envejecer el producto por un aproximado de $12 \mathrm{~h}$ a temperatura ambiente. El producto obtenido se filtró varias veces con agua ultrapura hasta llegar a neutralidad para luego ser llevado al vacío y posteriormente a la estufa a $150^{\circ} \mathrm{C}$. 


\section{Caracterización de los materiales}

Las propiedades magnéticas de los materiales fueron analizados mediante un magnetómetro de muestra vibrante (VSM), construido en el Laboratorio de Materiales Nanoestructurados de la Univesidad Nacional de Ingeniería, Perú. Los espectros de infrarrojo con transformada de Fourier (FTIR) fueron obtenidos usando un espectrómetro de reflectancia total atenuada (ATR, IR-Prestige 21, Shimadzu, Europa) en el rango de 4000 a $400 \mathrm{~cm}^{-1}$. Asimismo, los grupos funcionales presentes en los diversos materiales sintetizados fueron obtenidos a partir del espectro Raman utilizando un espectrómetro Raman (XploRA, HORIBA Scientific, Francia) con láser de 532 o $785 \mathrm{~nm}$. La morfología y microestructuras de la superficie de los materiales sintetizados y perlas preparadas fueron examinados utilizando el microscopio electrónico de barrido (SEM EVO MA10, Zeiss, Alemania). Las medidas voltamperométricas se realizaron con un potenciostato/galvanostato PalmSens ${ }^{\circledR}$ y una celda convencional de tres electrodos: electrodo de trabajo: carbón vítreo, electrodo auxiliar: alambre de platino y electrodo de referencia: electrodo $\mathrm{Ag} / \mathrm{AgCl}$ saturado con $\mathrm{KCl}$. Como electrolito se utilizaron buffer de fosfato.

\section{Pruebas de remoción de metales pesados usando el compósito Fe304/MWCNT/HAp} Para este caso, se escogió al As(V) dado que es uno de los metales pesados más contaminantes y de actual interés en la comunidad científica.

La remoción del As(V) se realizó utilizando como adsorbente al compósito de Fe3O4/ MWCNT/HAp. Esto se debe a que la hidroxiapatita mejora la preferencia de un compuesto a la adsorción de metales y la magnetita le conferiría la capacidad de recuperación y un posible reuso $^{18}$.

Se evaluó el efecto de $\mathrm{pH}$, para lo cual se utilizó una solución de $\mathrm{As}(\mathrm{V}) 1 \mathrm{mg} / \mathrm{L}$ en un rango de $\mathrm{pH}$ de 6 a 11, a las cuales se le añadió $10 \mathrm{mg}$ de compósito y se dejó en agitación por 4 h, pasado este tiempo se midió la concentración remanente. Se evaluó el efecto de tiempo de contacto, para lo cual se utilizó $20 \mathrm{~mL}$ de una solución de arsénico $1 \mathrm{mg} / \mathrm{L}$ y se le añadió 10 mg de compósito, se dejó agitando durante 4 h y luego se midió la concentración remanente. Se evaluó el efecto de masa de adsorbente, para lo cual se trabajó con $20 \mathrm{~mL}$ de una solución de arsénico $1 \mathrm{mg} / \mathrm{L}$ y se agregó 1,5; 6,2; 10,9; 15,6 y 22,1 mg de compósito y se dejó agitando durante $4 \mathrm{~h}$.

Las concentraciones iniciales y remanente (en todas las pruebas) fueron medidas utilizando un espectrofotómetro de absorción atómica (AA). Luego de cada proceso de remoción realizado, los materiales fueron recuperados con un imán mediante procesos de decantación. 


\section{RESULTADOS Y DISCUSIÓN}

\section{Caracterización de magnetita}

Al utilizar la técnica de dispersión dinámica de luz (DLS) para la magnetita estabilizada con citrato, se obtuvieron partículas con un diámetro efectivo de $113,9 \mathrm{~nm}$ y una polidispersidad de 0,229 (figura 1).

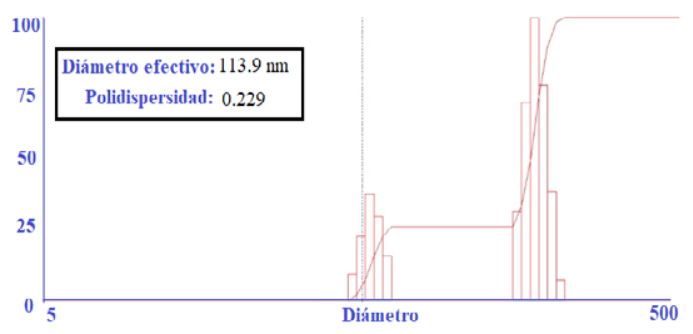

Figura 1. DLS de la magnetita sintetizada y recubierta con citrato.

La nanomagnetita estabilizada (Fe3O4-citrato) fue caracterizada mediante espectroscopía Raman (figura 2), mediante la cual se pudo obtener sus principales picos característicos: a 219 y $281 \mathrm{~cm}^{-1}$ los picos asociados al enlace Fe-O $\left(\mathrm{T}_{2 \mathrm{~g}}\right)$, a $398 \mathrm{~cm}^{-1}$ el pico asociado al OHpresente en la superficie del material, a $601 \mathrm{~cm}^{-1}$ el pico asociado al $\mathrm{Fe}-\mathrm{O}\left(\mathrm{A}_{\mathrm{gg}}\right)$ y finalmente el pico más intenso a $1311 \mathrm{~cm}^{-1}$ asociado al magnón. Por otra parte, se realizó la caracterización de $\mathrm{Fe}_{3} \mathrm{O}_{4}$ mediante magnetometría de muestra vibrante, obteniéndose la curva mostrada en la figura 2, la cual indica que el material obtenido muestra un comportamiento ferromagnético ${ }^{19}$ y de la cual se obtuvo además los siguientes parámetros: magnetización de saturación (Ms) de 64,2 $\mathrm{Am}^{2} \mathrm{~kg}^{-1}$, coercitividad cero y magnetización remanente (Mr) de 9,81 $\mathrm{Am}^{2} \mathrm{~kg}^{-1}$.
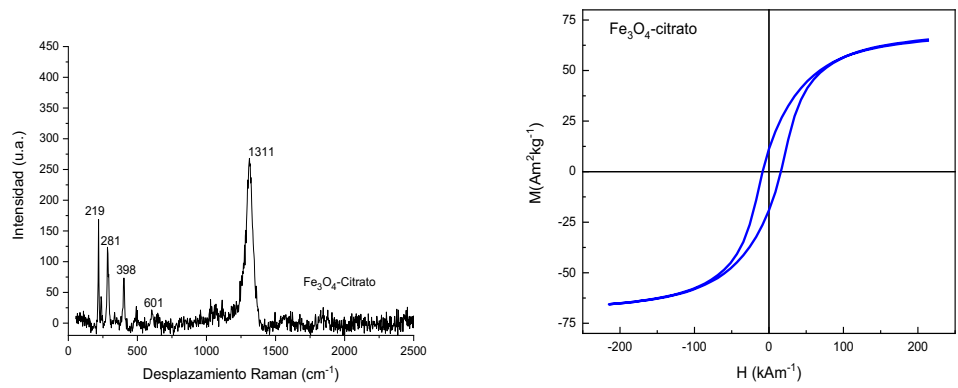

Figura 2. Espectro Raman (izquierda) y curva de magnetización (derecha) de magnetita estabilizada con citrato $\left(\mathrm{Fe}_{3} \mathrm{O}_{4}\right.$-citrato).

La magnetita-citrato también fue caracterizada mediante voltamperometría de separación anódica de onda cuadrada (figura 3), obteniéndose dos picos a -0,24 y 0,64 V, los cuales son característicos de la magnetita ya que representan la reducción de dos sitios cristalinos: uno tetraédrico (ocupado por $\mathrm{Fe}^{+3}$ ) y otro octaédrico (ocupado por $\mathrm{Fe}^{+2}$ y $\mathrm{Fe}^{+3}$ en cantidades 
iguales). Además, la muestra de magnetita sintetizada fue caracterizada mediante microscopía electrónica de barrido (figura 3), donde se observan cristales con poca aglomeración debido al uso de citrato como agente estabilizante.
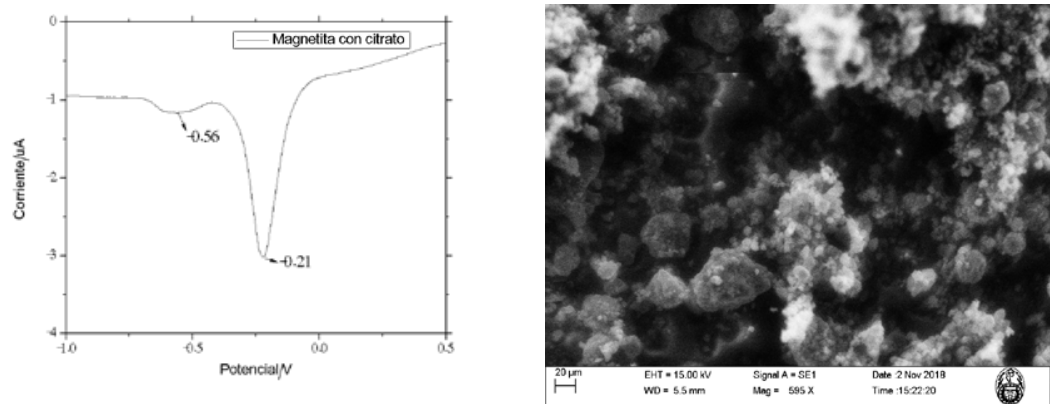

Figura 3. Curva de voltamperometría de onda cuadrada (izquierda) y micrografía SEM (derecha) de la magnetita-citrato sintetizada.

\section{Caracterización de hidroxiapatita}

Los tres tipos de hidroxiapatita sintetizadas (método químico, sol-gel e hidrotermal) fueron caracterizadas mediante espectroscopía Raman determinándose la presencia de sus principales picos característicos ${ }^{20}$. La figura 4 muestra los espectros Raman de la hidroxiapatita obtenida mediante método químico (método 1) y de la obtenida mediante método sol-gel (método 2), en ambos, se puede apreciar un pico intenso a $965 \mathrm{~cm}^{-1}$ el cual está asociado al modo $v 1$ del estiramiento totalmente simétrico del grupo $\mathrm{PO}_{4}$ (enlace $\mathrm{P}-\mathrm{O}$ ), también se aprecian dos picos de baja intensidad a 430 y $590 \mathrm{~cm}^{-1}$ asociados al modo de flexión $v 2,4$ de $\mathrm{PO}_{4}$ (enlace O-P-O), finalmente se aprecia un pico a $1020 \mathrm{~cm}^{-1}$ asociado al modo de estiramiento asimétrico 3 de PO4 (enlace P-O).

El espectro Raman de la hidroxiapatita obtenida mediante el método hidrotermal también se muestra en la figura 4, donde se destaca la presencia del pico a $961 \mathrm{~cm}^{-1}$.
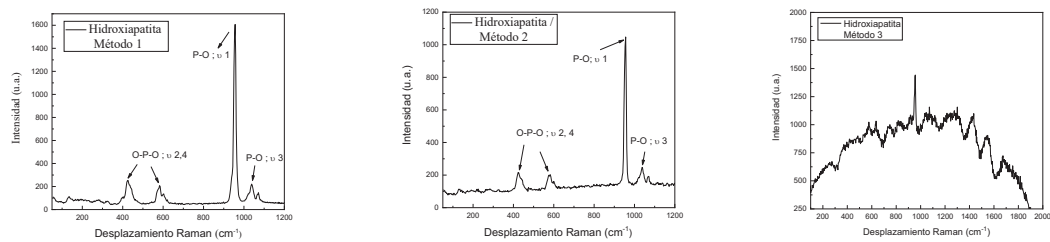

Figura 4. Espectros Raman de la hidroxiapatita (HAp) obtenida mediante el método 1, método 2 y método 3 .

La figura 5 muestra los resultados obtenidos al utilizar la técnica de dispersión dinámica de luz, mediante la cual se obtuvieron partículas de hidroxiapatita (método hidrotermal) con un diámetro efectivo de $103,4 \mathrm{~nm}$ y una polidispersidad de 0,244 , lo cual corresponde con lo reportado por la bibliografía ${ }^{16}$ de que se originan nanopartículas con un tamaño adecuado. 


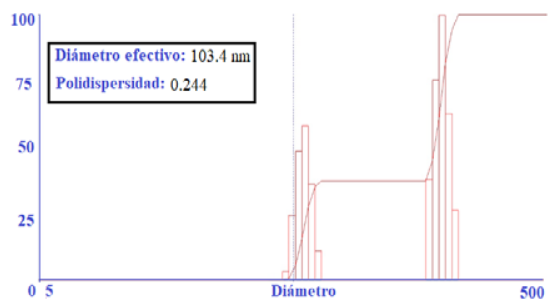

Figura 5. Espectros DLS de la hidroxiapatita (HAp) obtenida mediante el método 3.

\section{Caracterización del compósito $\mathrm{Fe}_{3} \mathrm{O}_{4} / \mathrm{HAp}$}

El compósito $\mathrm{Fe} 3 \mathrm{O} 4 / \mathrm{HAp}$ fue caracterizado mediante espectroscopía Raman (figura 6), en donde se observó que se mantiene el pico a $965 \mathrm{~cm}^{-1}$ asociado al enlace P-O (modo $v 1$ ) y el pico asociado al magnón se desplaza $\left(1393 \mathrm{~cm}^{-1}\right)$ y pierde ligeramente su forma.

Por otra parte, este compósito fue caracterizado mediante magnetometría de muestra vibrante (VSM). La curva muestra que se mantiene el comportamiento ferromagnético del material y que los valores parámetros de $\mathrm{M}_{\mathrm{s}}$ y $\mathrm{M}_{\mathrm{r}}$ disminuyen (en comparación de $\mathrm{Fe}_{3} \mathrm{O}_{4}$-citrato) a 6,25 y $1,25 \mathrm{Am}_{2} \mathrm{~kg}^{-1}$, respectivamente, lo cual se puede atribuir a la interacción de la magnetita con la hidroxiapatita, que es un material que no presenta propiedades magnéticas.

Este compósito también fue caracterizado mediante microscopía electrónica de barrido (SEM), en la cual se observó rugosidad ligera sobre la superficie del material.
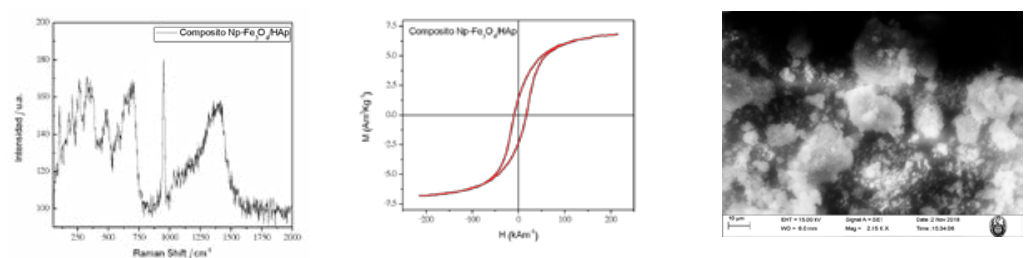

Figura 6. Espectro Raman, curva de magnetización y micrografías SEM del compósito $\mathrm{Fe}_{3} \mathrm{O}_{4}$-HAp.

\section{Caracterización del compósito $\mathrm{Fe}_{3} \mathrm{O}_{4} / \mathrm{MWCNT}$}

El compósito $\mathrm{Fe}_{3} \mathrm{O}_{4} / \mathrm{MWCNT}$ fue caracterizado mediante espectroscopía infrarroja (FTIR). La figura 7 muestra el espectro obtenido del compósito frente al espectro de los nanotubos, donde podemos observar que los picos asociados a los nanotubos se mantienen en el compósito y que destaca la presencia del pico a $690 \mathrm{~cm}^{-1}$, asociado al modo vibracional del Fe-O (uno de los picos característicos de la magnetita).

Por otra parte, el compósito fue caracterizado mediante microscopía SEM. La figura 8 muestra las micrografías de los nanotubos de carbono comerciales y del compósito Fe3O4/ MWCNT, en ellas podemos apreciar la aglomeración de los nanotubos (derecha) y una mayor dispersión de ellos en la formación del compósito (izquierda). 


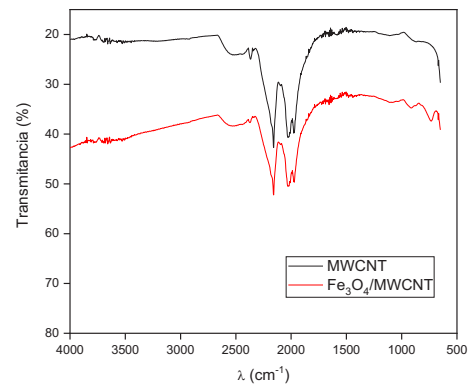

Figura 7. Espectros FTIR de los nanotubos de carbono (MWCNT) y el compósito $\mathrm{Fe}_{3} \mathrm{O}_{4} /$ MWCNT.
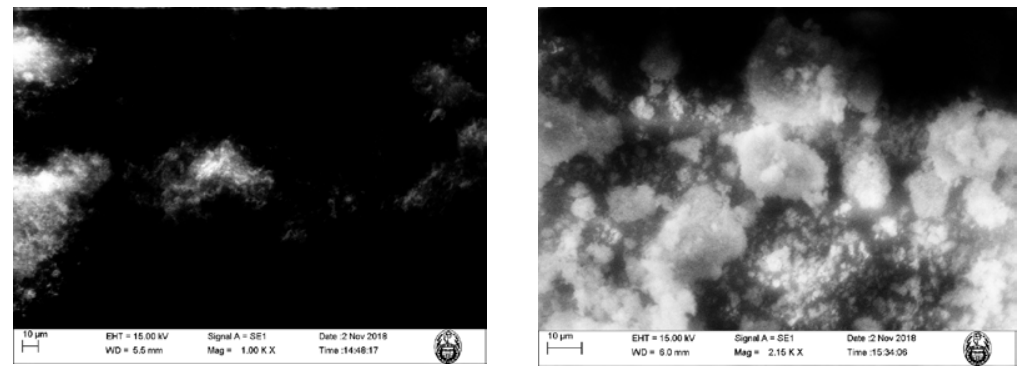

Figura 8. Imágenes SEM de los nanotubos de carbono (MWCNT) (derecha) y del compósito $\mathrm{Fe}_{3} \mathrm{O}_{4} / \mathrm{MWCNT}$ (izquierda).

\section{Caracterización del compósito Fe304/MWCNT/HAp}

El compósito $\mathrm{Fe}_{3} \mathrm{O}_{4} / \mathrm{MWCNT/HAp} \mathrm{también} \mathrm{fue} \mathrm{caracterizado} \mathrm{mediante} \mathrm{microscopía} \mathrm{SEM}$ (figura 9). En las figuras, se pudo observar la superficie rugosa del material, algunas regiones cavernosas, que podrían asociarse a la aglomeración del agente porogénico en el proceso de coprecipitación de la muestra.
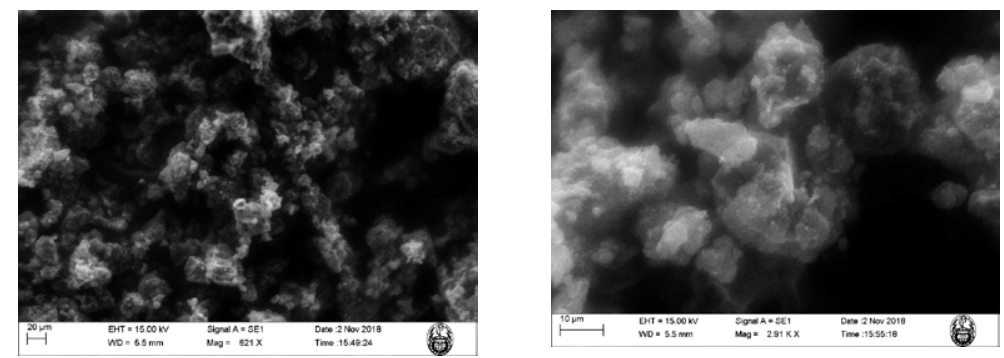

Figura 9. Imágenes SEM del compósito $\mathrm{Fe}_{3} \mathrm{O}_{4} / \mathrm{MWCNT} / \mathrm{HAp}$. 


\section{Pruebas de remoción de metales pesados \\ Efecto del pH}

Se eligió un rango de $\mathrm{pH}$ a partir de 6, en base a que lo reportado por la literatura, a partir de este valor se manifiesta una mejor eficiencia en el proceso de adsorción.

La figura 10 (izquierda) muestra una gráfica de porcentaje de remoción en función al pH. Se observó valores más altos porcentaje de remoción a un $\mathrm{pH} 6$ frente al resto de valores de $\mathrm{pH}$ probados $\left(7,8,10\right.$ y 11), lo cual coincide con lo reportado por trabajos anteriores ${ }^{21}$.

\section{Efecto de masa de adsorbente}

La figura 10 (derecha) muestra la gráfica de porcentaje de remoción en función a la masa de adsorbente, mediante la cual se obtuvo una relación directamente proporcional, donde el incremento de la masa de adsorbente, en nuestro caso, masa de compósito, generaba un aumento en los valores de la eficiencia de remoción. Además, se pudo notar que añadiendo desde 1,5 mg de adsorbente, los valores de porcentaje de remoción eran mayores al $80 \%$, indicando una alta remoción del metal. Por otra parte, fue posible observar que los valores de porcentajes de remoción obtenidos para 10,9; 15,6 y 22,1 mg fueron muy similares.
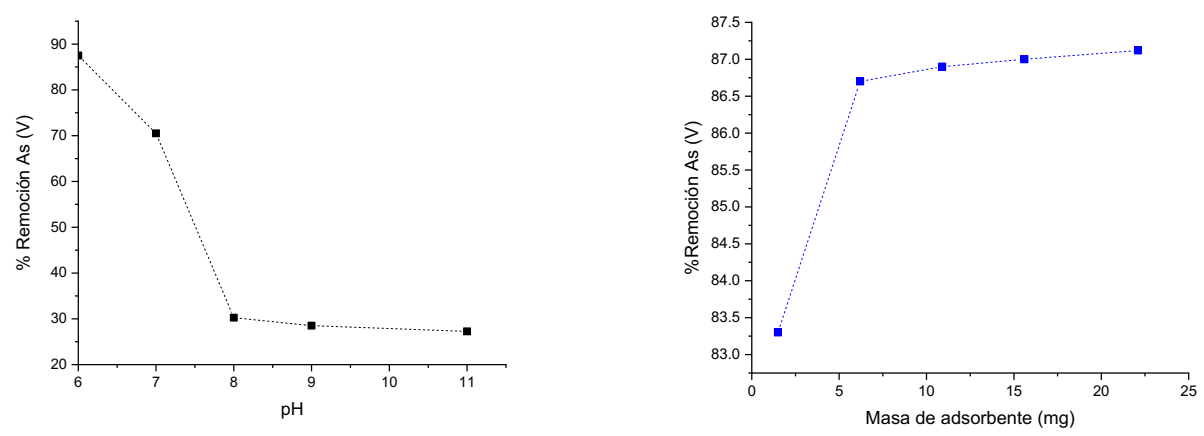

Figura 10. Gráfica de porcentaje de remoción en función a pH (izquierda) y masa de adsorbente (derecha).

\section{Efecto de tiempo de contacto}

La figura 11 muestra la gráfica de porcentaje de remoción en función al tiempo de contacto. Podemos observar que a medida que aumenta el tiempo de contacto, hay un incremento en la eficiencia de remoción. Sin embargo, la diferencia entre los valores de remoción obtenidos (tabla 1) no es muy marcada, por lo cual podríamos elegir, en base a lo obtenido, un tiempo de $2 \mathrm{~h}$ y el material nos estaría dando resultados eficientes. 


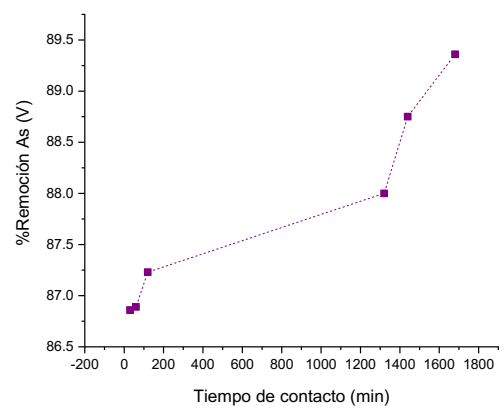

Figura 11. Gráfica de porcentaje de remoción en función al tiempo de contacto.

Tabla 1. Porcentaje de remoción de $\mathrm{As}(\mathrm{V})$ bajo diferentes tiempos de contacto.

\begin{tabular}{cc}
\hline $\begin{array}{c}\text { Tiempo de contacto } \\
\text { (min) }\end{array}$ & $\begin{array}{c}\text { \% Remoción de } \\
\mathbf{A s}(\mathbf{V})\end{array}$ \\
\hline 30 & 86,86 \\
\hline 60 & 86,89 \\
\hline 120 & 87,23 \\
\hline 1320 & 88,00 \\
\hline 1440 & 88,75 \\
\hline 1680 & 89,36
\end{tabular}

\section{CONCLUSIONES}

La magnetita $\left(\mathrm{Fe}_{3} \mathrm{O}_{4}\right)$, hidroxiapatita (HAp) y los compósitos $\left(\mathrm{Fe}_{3} \mathrm{O}_{4} / \mathrm{HAp}, \mathrm{Fe}_{3} \mathrm{O}_{4} / \mathrm{MWCNT}\right.$ y Fe3O4/MWCNT/HAp) fueron sintetizados y caracterizados mediante las técnicas de: DLS, VSM, SEM, FTIR, Raman y técnicas voltamperométricas. Se realizaron pruebas preliminares en la remoción de $\mathrm{As}(\mathrm{V})$ variando los parámetros de $\mathrm{pH}$, masa de adsorbente y tiempo de contacto. Se obtuvo los valores máximos de porcentaje de remoción de este metal (mayor a $85 \%$ ) a condiciones de $\mathrm{pH} 6$, dosis de adsorbente de $0,5 \mathrm{~g} \mathrm{~L}^{-1}$ y un tiempo de contacto de 2 $\mathrm{h}$, dándonos buenos indicios de hacer un estudio más amplio y con otros metales pesados.

\section{AGRADECIMIENTOS}

Los autores agradecen al Grupo de Investigación de Electroquímica Aplicada por brindar los equipamientos e instalaciones para la realización de los experimentos desarrollados y a la Oficina de Gestión de la Investigación de la Universidad Nacional de Ingeniería (OGI - UNI) por el financiamiento para la realización de la investigación. 


\section{REFERENCIAS BIBLIOGRÁFICAS}

1. Londoño-Franco L, Londoño-Muñoz P, Muñoz-García F. Los riesgos de los metales pesados en la salud humana y animal. Biotecnol Sector Agropecuario Agroind. 2017; 14(2): 145-152.

2. Goswami A, Purkait M, Removal of fluoride from drinking water using nanomagnetite aggregated schwertmannite. J Water Process Eng. 2014; 1: 91-100.

3. Rafiee E, Eavani S. $\mathrm{H}_{3} \mathrm{PW}_{12} \mathrm{O}_{40}$ supported on silica-encapsulated $\gamma-\mathrm{Fe}_{2} \mathrm{O}_{3}$ nanoparticles: a novel magnetically-recoverable catalyst for three-component Mannich-type reactions in water. Green Chem. 2011; 13:2116-2122.

4. Khedr MG. Nanofiltration and low energy reverse osmosis for rejection of radioactive isotopes and heavy metal cations from drinking water sources. Desalin Water Treat. 2009; 2: 342-350.

5. Tejada-Tovar C, Villabona-Ortiz Á, Garcés-Jaraba Luz. Adsorción de metales pesados en aguas residuales usando materiales de origen biológico. TecnoLógicas. 2015; 18(34): 109-123.

6. Iwahori K, Watanabe J, Tani Y, Seyama H, Miyata N. Removal of heavy metal cations by biogenic magnetite nanoparticles produced in $\mathrm{Fe}(\mathrm{III})$-reducing microbial enrichment cultures. J Biosci Bioeng. 2014;117(3):333-335.

7. Pan J, Wan D, Bian Y, Sun H, Zhang C, Jin F, et al. Fluorescent Hydroxyapatite-Loaded Biodegradable Polymer Nanoparticles with Folate Decoration for Targeted Imaging. Aiche J. 2013; 59: 4494-4501.

8. Zhang C, Li C, Huang S, Hou Z, Cheng Z, Yang P, et. al. Self-activated luminescent and mesoporous strontium hydroxyapatite nanorods for drug delivery. Biomaterials. 2010; 31: 3374-3383.

9. Szcześ A, Hołysz L, Chibowski E. Synthesis of hydroxyapatite for biomedical applications. Adv Colloid Interface Sci. 2017; 249:321-330.

10. Coreño AJ, Mújica CJM, Hernández CD, Herrera GAM, García SJ. Evaluación de hidroxiapatita nanoparticulada como material adsorbente de iones flúor, plomo y arsénico en soluciones acuosas. Superficies y Vacío. 2010; 23: 161-165.

11. Janovák L, Deák A, Tallósy SP, Sebok D, Csapó E, Bohinc K, et al. Hydroxyapatiteenhanced structural, photocatalytic and antibacterial properties of photoreactive $\mathrm{TiO}_{2} /$ HAp/polyacrylate hybrid thin films. Surf Coat Tech. 2017; 326: 316-326.

12. Zhu Y, Quan X, Chen F, Fan X, Feng Y. $\mathrm{CeO}_{2}-\mathrm{TiO}_{2}$ Coated Ceramic Membrane with Catalytic Ozonation Capability for Treatment of Tetracycline in Drinking Water. Sci Adv Mater. 2012; 4(12): 1191-1199.

13. López F, Alguacil F, Álvarez T, Cerpa A. Utilización de nanotubos de carbono para la eliminación de metales tóxicos en agua. [Internet] Madrid-España: Congreso Nacional del Medio Ambiente; 2014. [Citado 14 jul 2019]. Disponible en: http://www.conama11. vsf.es/conama10/download/files/conama2014/CT\%202014/1896711012.pdf

14. Sequeda L, Díaz J, Gutiérrez S, Perdomo S, Gómez O. Obtención de hidroxiapatita sintética por tres métodos diferentes y su caracterización para ser utilizada como sustituto óseo. Rev Colomb Cienc Quim Farm. 2012; 41(1): 50-66. 
15. Rivera JA, Fetter G, Bosch P. Efecto del pH en la síntesis de hidroxiapatita en presencia de microondas. Matéria. 2011; 15(4): 506-515.

16. Jin X, Zhuang J, Zhang Z, Guo H, Tan J. Hydrothermal synthesis of hydroxyapatite nanorods in the presence of sodium citrate and its aqueous colloidal stability evaluation in neutral pH. J Colloid Interface Sci. 2015; 443:125-130.

17. Iwasaki T, Nakatsuka R, Murase K, Takata H, Nakamura H, Watano S. Simple and rapid synthesis of magnetite/hydroxyapatite composites for hyperthermia treatments via a mechanochemical route. Int J Mol Sci. 2013;14(5): 9365-78.

18. Giraldo L, Erto A, Moreno-Pirajan JC. Magnetite nanoparticles for removal of heavy metals from aqueous solutions: synthesis and characterization. Adsorption. 2013; 19(24): 465-474.

19. Li YS, Church JS, Woodhead AL. Infrared and Raman spectroscopic studies on iron oxide magnetic nano-particles and their surface modifications. J Magn Magn Mater. 2012; 324:1543-1550.

20. Koutsopoulos S. Synthesis and characterization of hydroxyapatite crystals: a review study on the analytical methods. J Biomed Mater Res. 2002; 62(4):600-612.

21. Martínez C, Aguilera E, Martínez A. Remoción de arsénico de agua potable mediante adsorción sobre óxido y oxihidróxidos de hierro. Ciencia Cierta. 2018; 56: 1-14. 